Original Research Paper

\title{
Genetic Diversity and Drug Resistance of Mycobacterium tuberculosis Complex Isolates and Nontuberculous Mycobacteria Identification from Presumptive Tuberculosis Cases in Oaxaca, Mexico
}

\author{
1,a,b Nakamura-Lopez Yuko, ${ }^{1,2}$ Valencia-Carmona Oscar Daniel, ${ }^{2,6}$ Martinez-Cruz Perla Monica, \\ ${ }^{3}$ Palma-Nicolas Jose Prisco, ${ }^{4, a, b}$ Gonzalez-y-Merchand Jorge Alberto, ${ }^{4}$ Rivera-Gutierrez Sandra, \\ 5,a,b Muñiz-Salazar Raquel and ${ }^{2}$ Martinez-Martinez Lucia Lourdes
}

\author{
${ }^{1}$ Laboratorio de Biología Molecular, Consejo Estatal para la Prevencion y Control del Sida. San Bartolo Coyotepec, \\ Oaxaca, Mexico \\ ${ }^{2}$ Laboratorio de Biologia Molecular, Centro de Investigación, Facultad de Medicina UNAM-UABJO, \\ Universidad Autonoma "Benito Juarez" de Oaxaca, Oaxaca, Mexico \\ ${ }^{3}$ Laboratorio de Micobacterias, Centro Regional de Control de Enfermedades Infecciosas, \\ Facultad de Medicina, Universidad Autonoma de Nuevo Leon, Monterrey, Nuevo Leon, Mexico \\ ${ }^{4}$ Departamento de Microbiologia, Escuela Nacional de Ciencias Biologicas, \\ Instituto Politecnico Nacional, Ciudad de Mexico, Mexico \\ ${ }^{5}$ Laboratorio de Epidemiología y Ecología Molecular, Escuela de Ciencias de la Salud, \\ Universidad Autónoma de Baja California, Ensenada, Baja California, México \\ ${ }^{6}$ Division de Estudios de Posgrado e Investigacion, Instituto Tecnologico de Oaxaca, Oaxaca, Mexico \\ ${ }^{a}$ Red Multidisciplinaria de Investigación en Tuberculosis (RemiTB; www.remitb.org) \\ ${ }^{b}$ Red Nacional de Investigación para la Prevención y Control de la Tuberculosis en Mexico
}

\section{Article history}

Received: 08-10-2019

Revised: 17-12-2019

Accepted: 04-02-2020

Corresponding Author:

Nakamura-Lopez Yuko

Laboratorio de Biología

Molecular, Consejo Estatal para la Prevencion y Control del Sida. San Bartolo Coyotepec, Oaxaca, Mexico

Email: nakamura.yuko@gmail.com

\begin{abstract}
Tuberculosis (TB) is a re-emerging health problem worldwide. In Mexico, information about genetic diversity and drug resistance of Mycobacterium Tuberculosis complex (MTBc) is limited. Samples from 463 Presumptive TB cases were tested for TB by smear, culture and PCR, from which $19.2 \%$ were identify as $\mathrm{MTBc}$ and $16 \%$ as nontuberculous mycobacteria. MTBc isolates were characterized by Large Sequence Polymorphisms (LSPs), spoligotyping and MIRU-VNTR 24 loci typing methods. Clade designations showed 10 sub-lineages: Haarlem $(35.7 \%)$, EAI (26.2\%), LAM (9.5\%), Ghana (4.8\%), X (2.4\%), New-1 (2.4\%), H37Rv-like (7.1\%), M. bovis (2.4\%), S (7.1\%), Uganda I (2.4\%). The finding of EAI as one of the principal genotypes may be associated with high migration rates. Drug resistance was found in $35.71 \%$ of the isolates: $14.2 \%$ were multidrug-resistant (MDR-TB), $14.2 \%$ mono-resistant and $7.14 \%$ poly-resistant. This study provides the first description of genetic diversity and drug resistance profile of MTBc in Oaxaca, Mexico.
\end{abstract}

Keywords: Mycobacterium tuberculosis Complex, Genotyping, Tuberculosis

\section{Introduction}

Tuberculosis (TB) is considered since 1993 by World Health Organization as an emergent public health worldwide disease. In 2014, 9.6 million people developed TB and 1.5 million died by this disease (WHO, 2015). Mexico ranks third in TB cases in the Americas (OPS/OMS, 2014) and the state of Oaxaca has incidence and mortality rates above the national average (PUDI, 2014). According to ethnic and geographical criteria, Oaxaca is divided in eight geocultural regions with a high level of indigenous population and, in addition, immigration and emigration rates (nationally and internationally) are the highest among many other states of the south of Mexico. In Oaxaca, about 3.9 million people demand healthcare attention (PUDI, 2014) and 500-700 new TB cases were reported each year in the last decade. 
Genotyping of Mycobacterium tuberculosis complex $(\mathrm{MTBc})$ is a powerful tool that helps, at individual and population level to the disease management (Coll et al., 2014), however there is no information about the TB genotypic characteristics from Oaxaca. The inclusion of a few number of clinical isolates from this region in previous studies suggest that genotypic composition of circulating strains from the Southwest States (Guerrero, Oaxaca and Chiapas) differ from the rest of the country (Nava-Aguilera et al., 2011), but not conclusive data was previously available about Oaxaca. At the same time, the prevalence of drug resistance or MDR strains in Oaxaca was under represented in recent works (Macias Parra et al., 2011; Martinez-Guarneros et al., 2013). The genotyping methodologies more widely used due to its traceability, robustness and uniformity in the interpretation of results are spoligotyping, MIRU-VNTR and LSP (Allix et al., 2008; Reed et al., 2009; Supply et al., 2006).

In this work, we used the methodologies mentioned above to determine the first insight into the genetic diversity, drug resistance and comorbidities of MTBc isolates circulating in a southwest state of Mexico.

\section{Materials and Methods}

\section{Sample Collection}

Pulmonary and extra-pulmonary samples (sputum, lymph node biopsies, cerebrospinal fluids and bronchoalveolar lavages), were obtained from people with suspect of tuberculosis during the period 2014-2015 throughout Oaxaca State regions (Papaloapan, Cañada, Costa, Istmo, Mixteca, Sierra Norte, Sierra Sur and Valles Centrales (Fig. 1)).

\section{Mycobacterial Identification and Drug Susceptibility Testing}

Sputum samples were collected and decontaminated following the modified Petroff's method (Petroff, 1915). Mycobacteria isolation was carried out in Löwenstein-Jensen (LJ) medium. Characterization of AFB (+) isolates was achieved by phenotypic tests, macroscopic morphology of colonies, growth speed, niacin (+) and nitrite production (Campos and Flores, 1996; Huard et al., 2003). All clinical samples were manipulated under biosafety conditions using a Biological Safety Cabinet IIA in a BSL-2 facility.

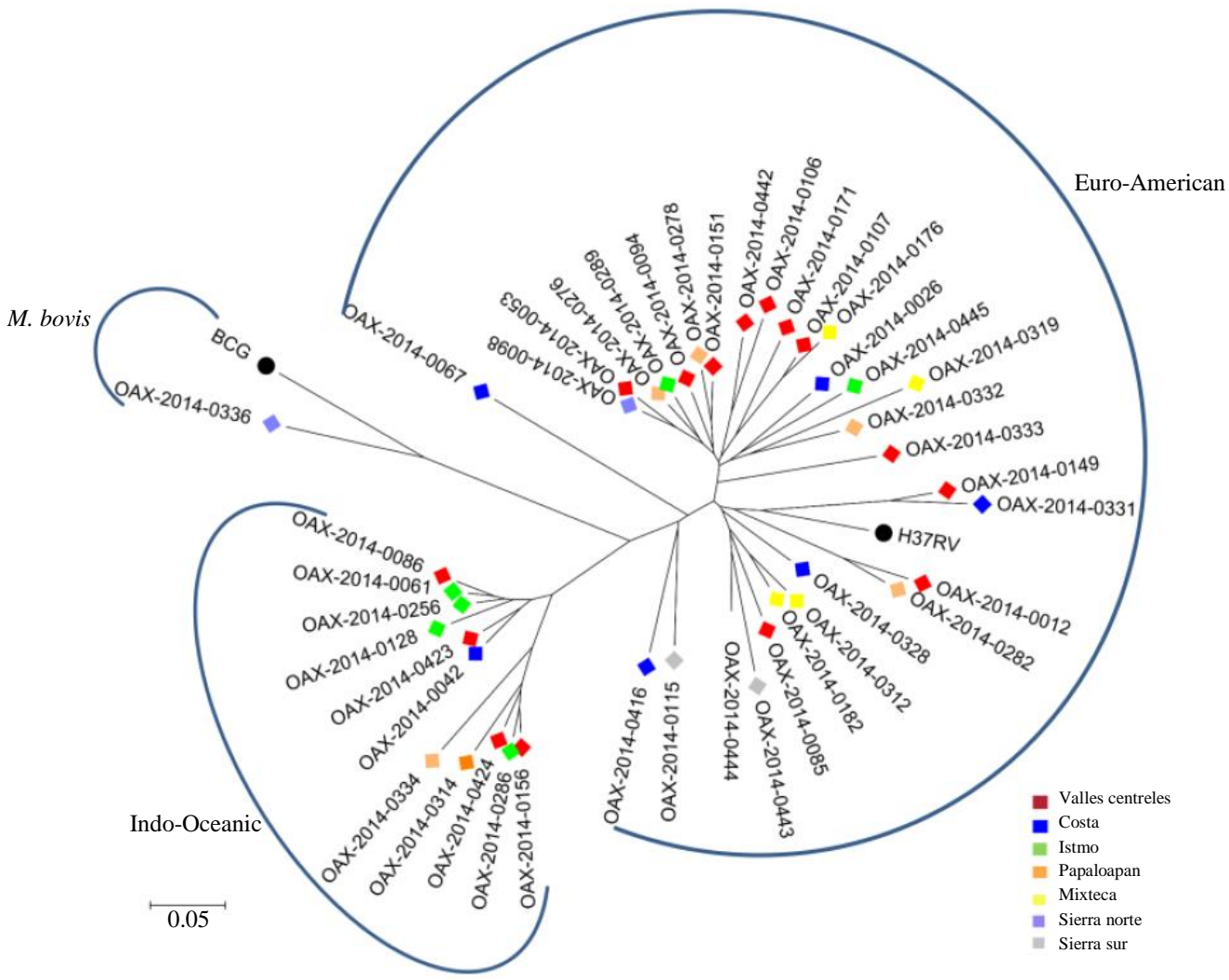

Fig. 1: Radial phylogenetic tree based on LPS, MIRU-VNTR and spoligotyping. The principal genetic lineage of each cluster is indicated and the geographical region where the isolates belonged to: Valles Centrales (red square), Costa (blue square), Istmo (green square), Papaloapan (orange square), Mixteca (yellow square), Sierra Norte (purple square) and Sierra Sur (gray square). Reference strains included M. tuberculosis H37Rv and M. bovis BCG (black circles) 
Mycobacterial identification was done by Gene Xpert MTB/RIF (Cepehid, USA) according to manufacturer's specifications and by PCR using specific primers as described previously (Huard et al., 2003). Drug resistance was determined by the indirect proportion method on LJ medium at critical concentrations for Isoniazide (INH; $0.2 \mu \mathrm{g} / \mathrm{mL}$ ), Rifampin (RIF; 40 $\mu \mathrm{g} / \mathrm{mL})$, Streptomycin (STR; $4.0 \mu \mathrm{g} / \mathrm{mL})$ and Ethambutol (EMB; $2.0 \mu \mathrm{g} / \mathrm{mL}$ ) (Workneh et al., 2016). Pyrazinamidase activity was used to determine susceptibility to Pyrazimide (PZA).

\section{Genotyping}

DNA of each clinical isolate was extracted using a commercial kit (DNAzol, Thermo Fisher Scientific, USA) and was quantified by UV spectrophotometry (UV Spectrophotometer Q3000, Quawell, USA). MTBc clinical isolates were genotyped by MIRUVNTR 24loci (Allix et al., 2008), spoligotyping (Supply et al., 2006) and LSP including the regions IS1561, RD4, RD9, RD12 (Huard et al., 2003), RD105, RD239, RD750 (Reed et al., 2009), RD724 and RD726 (Rindi et al., 2014).

PCR products were evaluated by electrophoresis on agarose gels stained with ethidium bromide and by Bioanalyzer DNA chip (Agilent, Santa Clara, CA). $M$. tuberculosis $\mathrm{H} 37 \mathrm{Rv}$ and $M$. bovis BCG were used as reference strains.

\section{Bioinformatic Analysis}

Spoligotype patterns as binary format, 24-loci MIRU patterns and presence/absence of RDs were analyze using the MIRU-VNTR plus database (http://www.miruvntrplus.org) to determine the genotypes and the matrix of distances. MEGA6 software (MEGA software) was used to build the phylogenetic tree using the neighborjoining algorithm.

\section{Results}

\section{Mycobacterial Isolation and Drug Susceptibility Testing}

From January 2014 to March 2015, 463 individuals with presumptive Mycobacterium tuberculosis infection of the eight regions of Oaxaca were tested. Some $21 \%$ samples were positive by PCR/XPERT and $16 \%$ were positive to Nontuberculous Mycobacteria (NTM). The samples from "Cañada" region were negative for MTBc nevertheless positive to NTM.

We recovered 42 clinical isolates of $\mathrm{MTBc}$, all from 2014. Two isolates $(4.7 \%)$ were recovered from extrapulmonar location (lymph node and cerebrospinal) and the rest were from respiratory samples. According to gender, $67 \%$ TB patients were men and $33 \%$ were women, all of them were diagnosed/treated as new TB cases. The most frequent comorbidities found were type 2 Diabetes Mellitus (DM) (33.3\%), malnutrition (21.4\%) and HIV coinfection (11.9\%).

From $42 \mathrm{AFB}(+)$ cultures, one niacin (-) and nitrite (-) isolate was identified as Mycobacterium bovis with intrinsic Pyrazinamide (PZA) resistance, while 15 $(35.7 \%)$ isolates showed resistance to at least one drug. As shown in Table 1, one isolate $(2.38 \%)$ was RIF mono-resistant and three $(7.1 \%)$ were STR monoresistant. MDR-TB strains recovered in this study account for $14.3 \%$ (6) of all isolates.

\section{Genotyping Profiles of MTBc Strains}

Using MIRU-VNTR 24 loci, spoligotyping and LSP, $42 \mathrm{MTBc}$ isolates were analyzed. Considering data of the three markers, a distance matrix was calculated and a phylogenetic tree was constructed using the neighbor-joining algorithm. The isolates were classified into three major lineages: Indo-Oceanic, Euro-American and M. bovis (Fig. 1).

As shown in Table 1, the Euro-American was the principal genetic lineage represented with thirty isolates, including 13 Haarlem (30.9\%), 4 LAM (9.5\%), 5 H37Rv-like (11.9\%) sub-lineages, accounting for $52 \%$ of all isolated strains. The second principal genetic lineage identified was the Indo-Oceanic with eleven (26.1\%) M. tuberculosis isolates of EAI sublineage, which was also found highly associated to first-line drug resistance with five $(45 \%)$ isolates being resistant to at least one drug (Table 1).

The frequency of other minor genotypes and their association with drug resistance is indicated in Table 1. It is noteworthy the high burden of EAI sub-lineage in Oaxaca and the high genetic diversity of the strains belonging to the Euro-American lineage.

The sub-lineages identified were located in seven of the eight geographical regions that conform the state of Oaxaca (Fig. 2). The "Valles Centrales" and "Costa" regions showed the highest diversity of lineages, with six and five sub-lineages respectively. In the "Valles Centrales" Euro-American Haarlem sub-lineage was the first most frequent (33.3\%), followed by Indo-Oceanic EAI sub-lineage (26.6\%) accounting $60 \%$ of the isolates; only one EAI isolate showed a monodrug resistance pattern to STR. In the "Costa" region, $66.6 \%$ of the isolates showed antituberculosis drug resistance, the $\mathrm{S}$ sub-linage was the most frequent $(33.3 \%)$, one of this strains was resistant to all the anti-tuberculosis drugs tested and another was MDR-TB. 
Nakamura-Lopez Yuko et al. / American Journal of Infectious Diseases 2020, 16 (1): 7.19 DOI: 10.3844/ajidsp.2020.7.19

Table 1: Demographic, genotyping data and drug resistance pattern of $42 \mathrm{MTBc}$ isolates

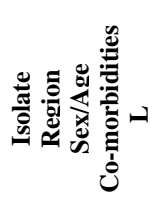

$$
\text { MIRU24 }
$$

LSP

Spoligotyping

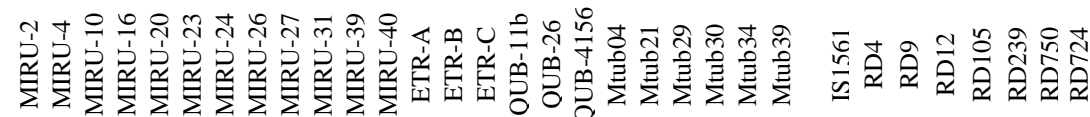

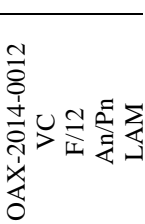

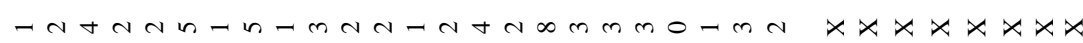

$$
\text { 感 }
$$

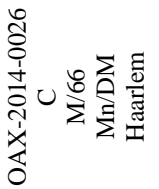

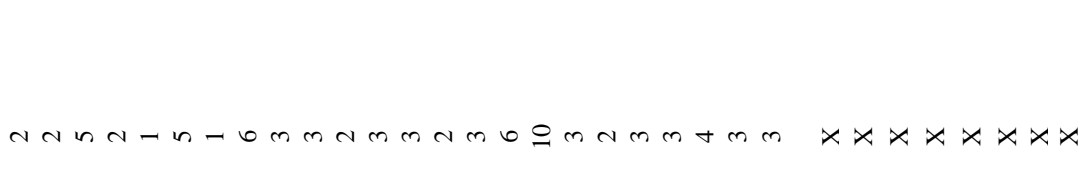

$\stackrel{8}{8}$

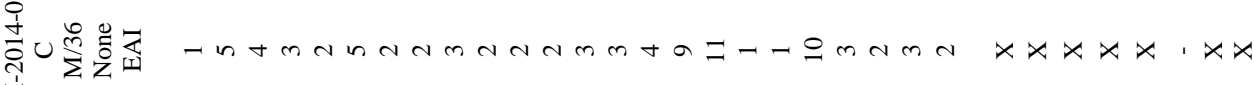

离

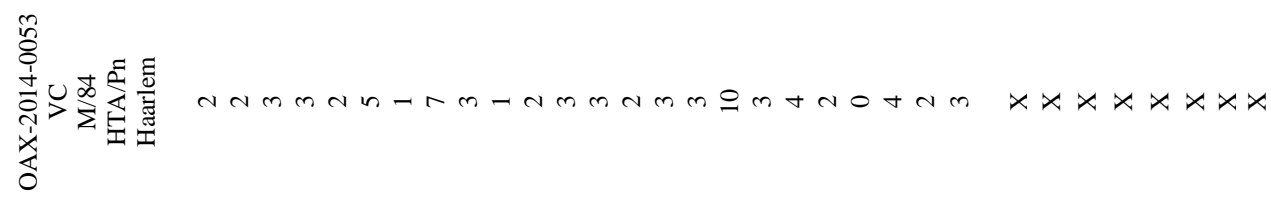

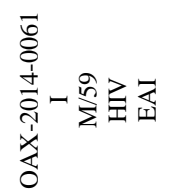

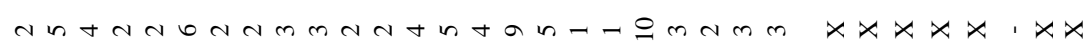

这

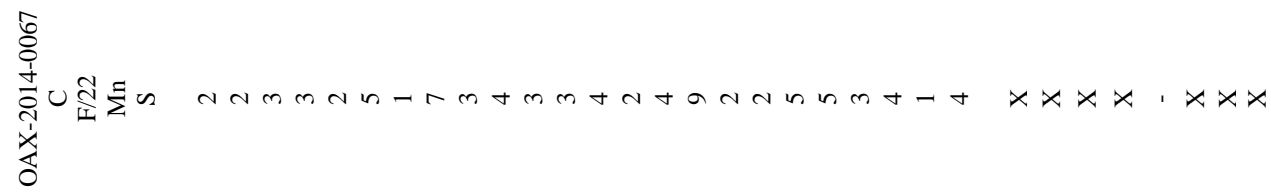

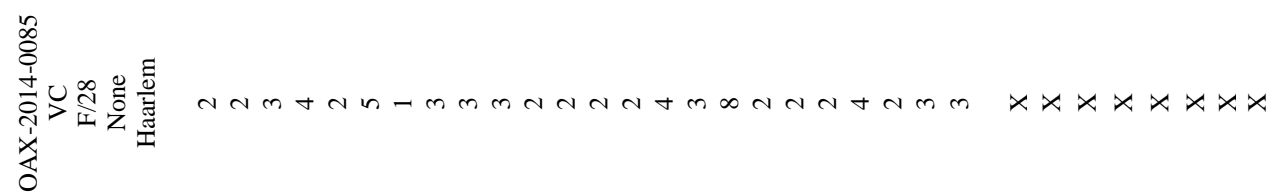

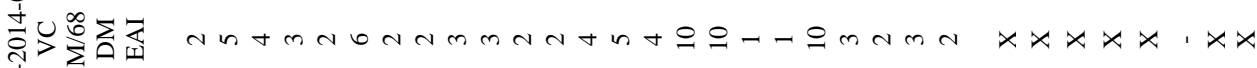


Nakamura-Lopez Yuko et al. / American Journal of Infectious Diseases 2020, 16 (1): 7.19 DOI: 10.3844/ajidsp.2020.7.19

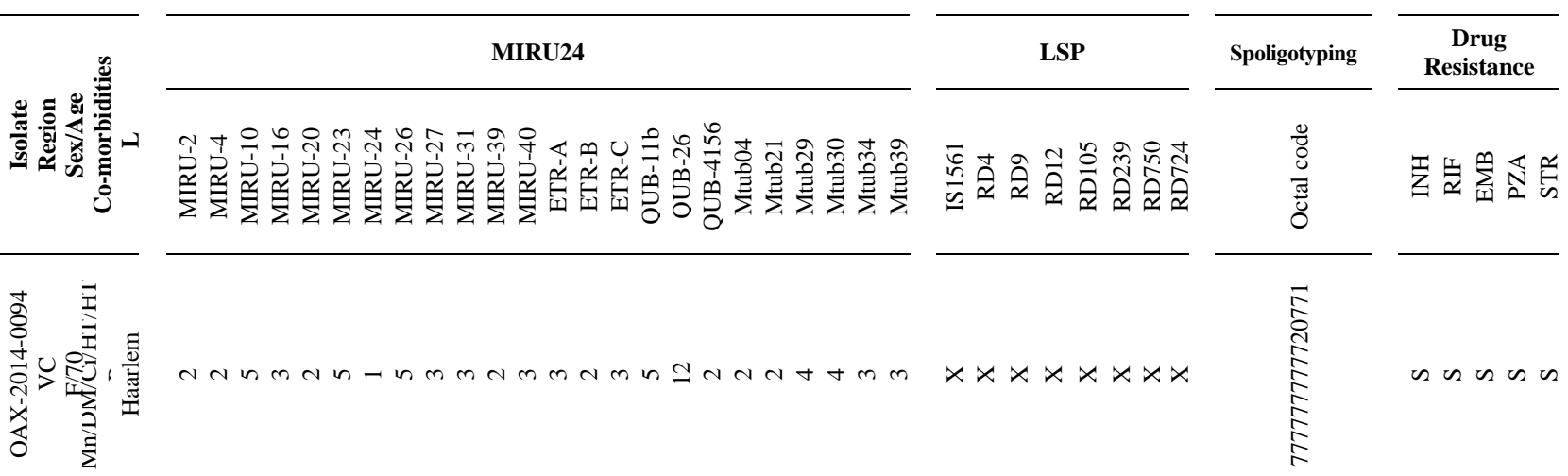

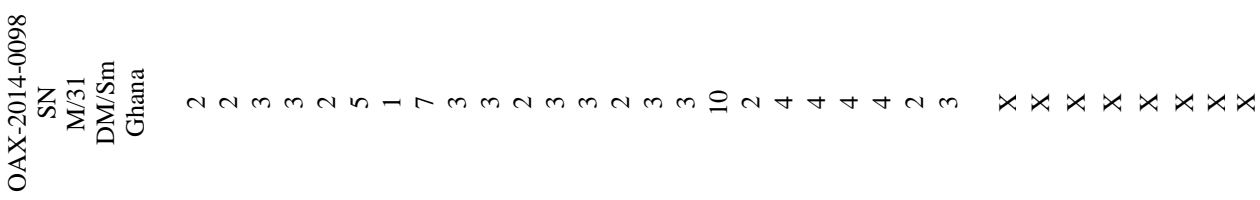

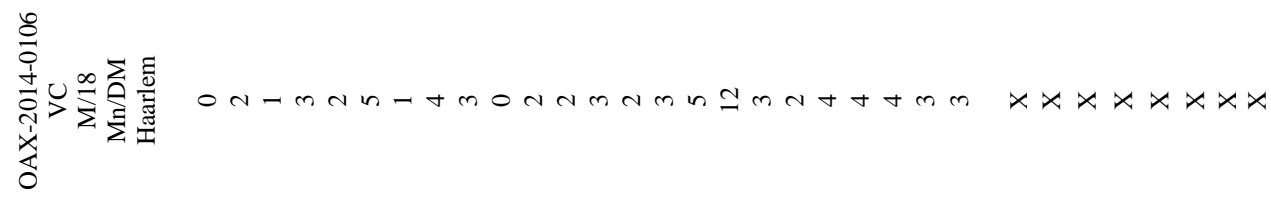

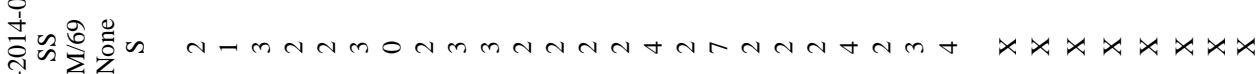
峁

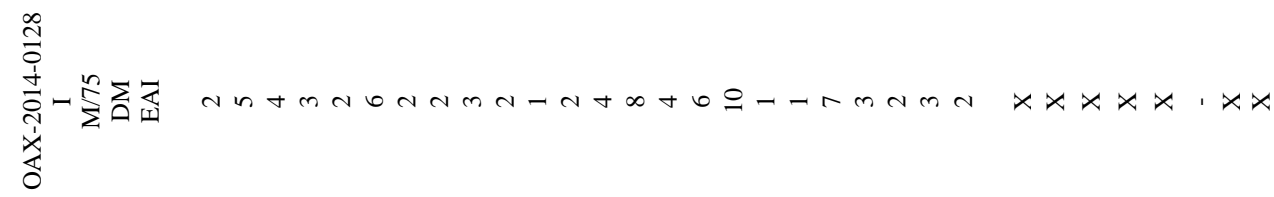

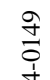

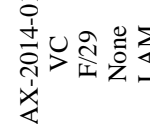

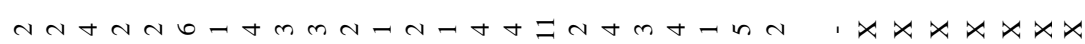

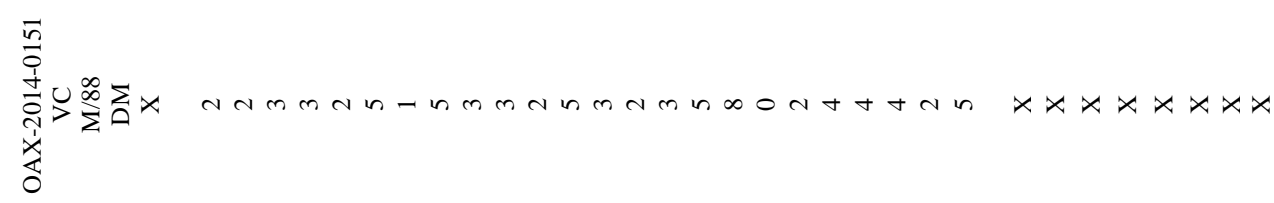


Nakamura-Lopez Yuko et al. / American Journal of Infectious Diseases 2020, 16 (1): 7.19 DOI: 10.3844/ajidsp.2020.7.19

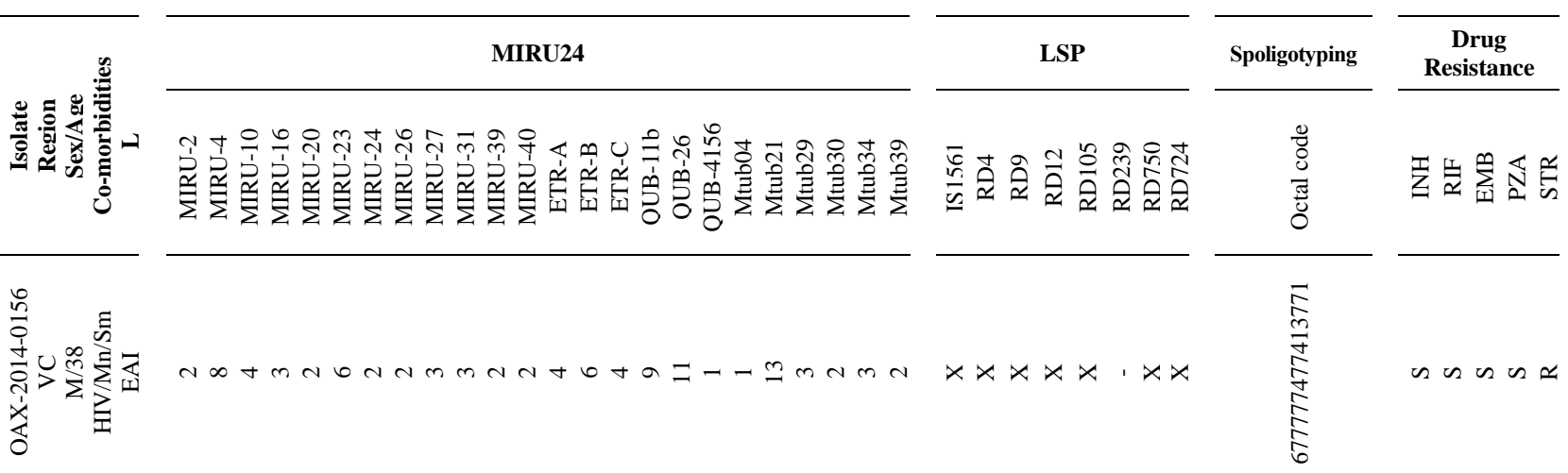

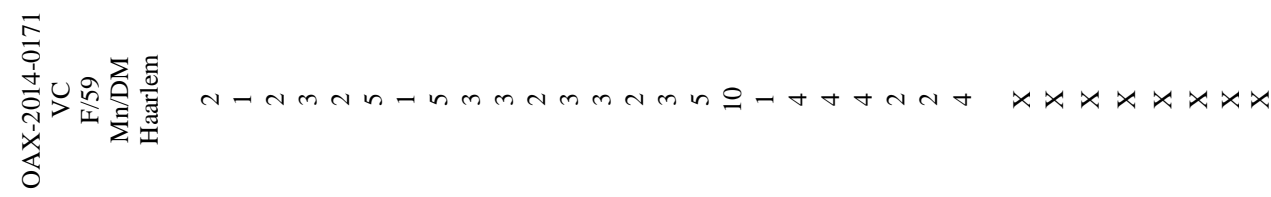

$\stackrel{\infty}{\infty}$

iั

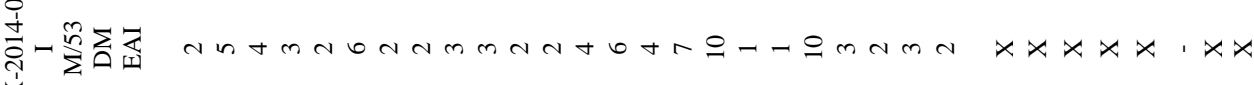


Nakamura-Lopez Yuko et al. / American Journal of Infectious Diseases 2020, 16 (1): 7.19 DOI: 10.3844/ajidsp.2020.7.19

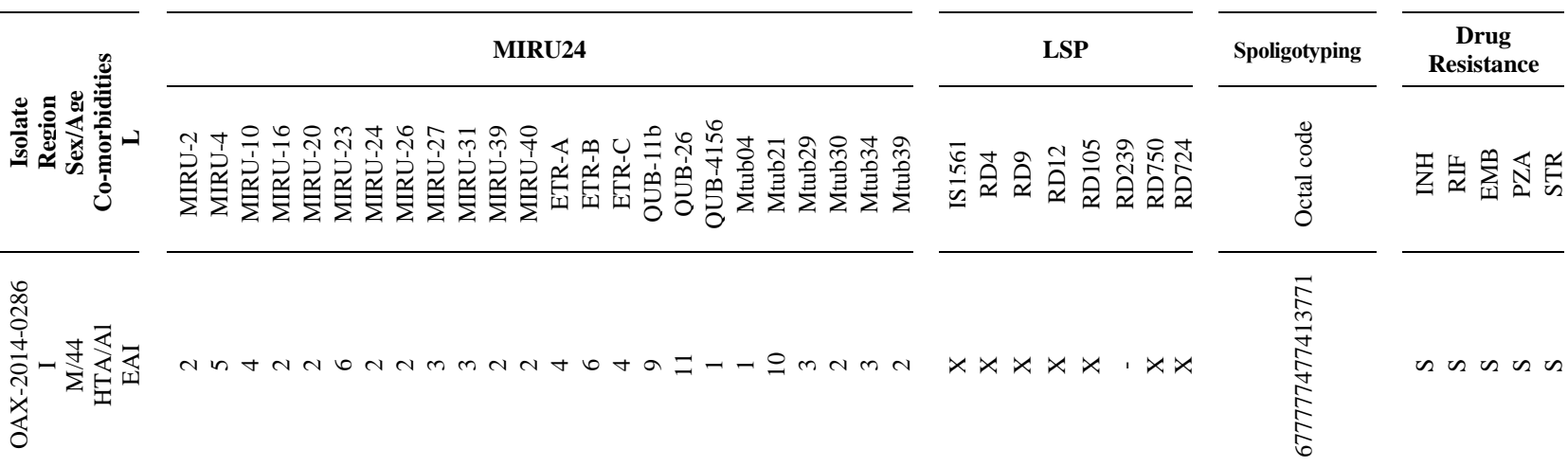

㖉

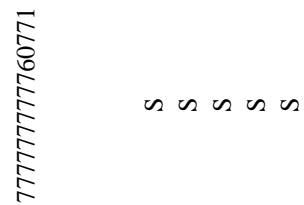

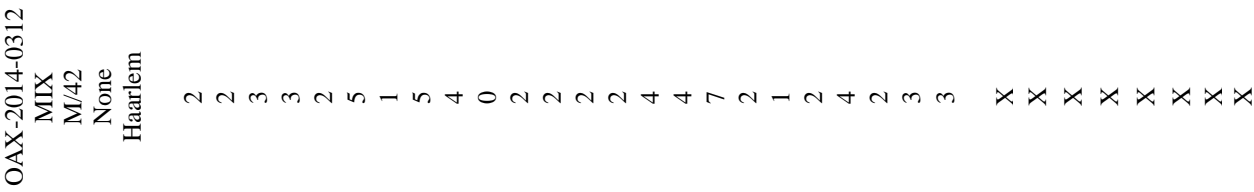

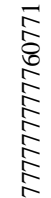

un un u

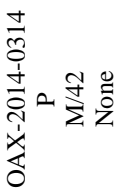

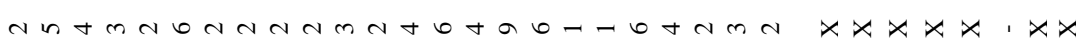

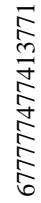

as un us

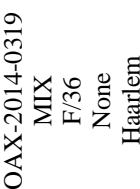

옹

in in in is

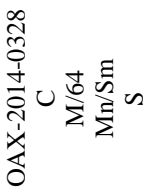

$4 a m m a n-m+m n t m n \operatorname{tat} n m n t-m+x x x x x x x$

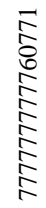

on $n$ \& $n$

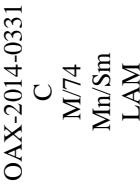

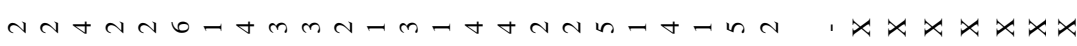

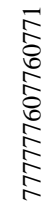

os os n o $\simeq$

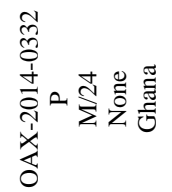

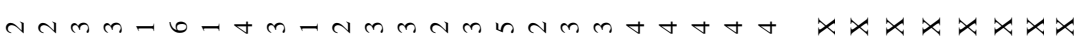


Nakamura-Lopez Yuko et al. / American Journal of Infectious Diseases 2020, 16 (1): 7.19 DOI: 10.3844/ajidsp.2020.7.19

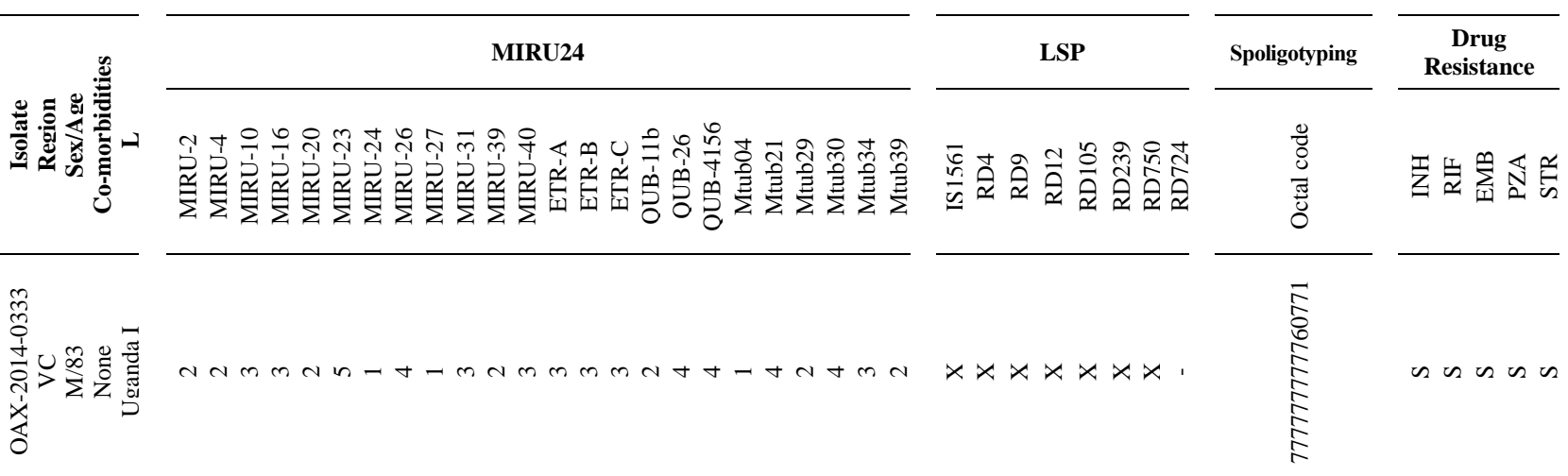

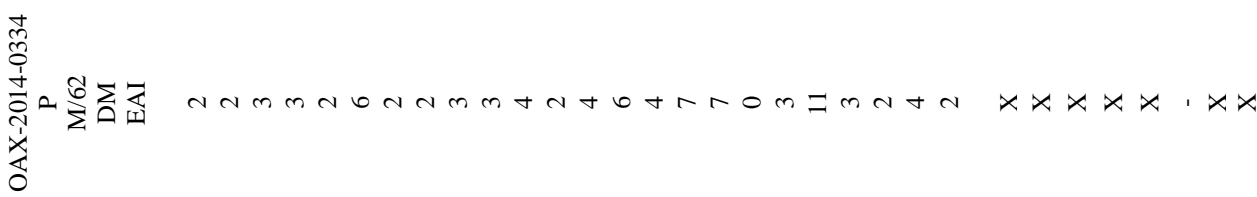

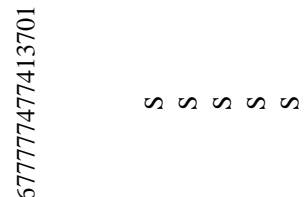

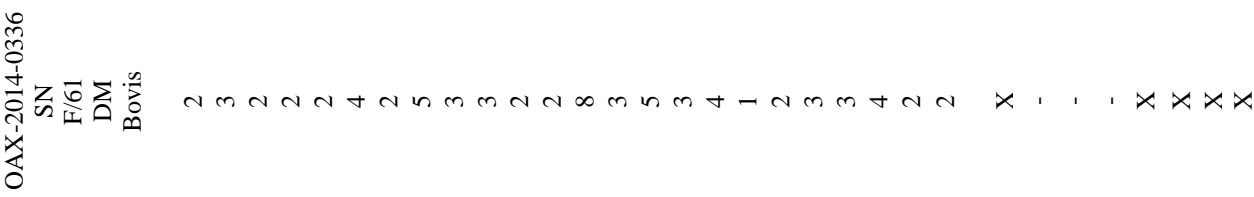

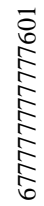

$\infty \cos \simeq$ a

焉

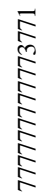

un as n a

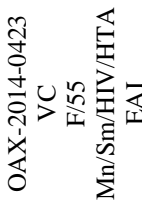

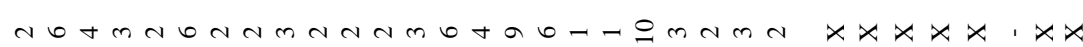

除

in $n$ \& $n$

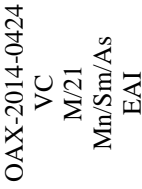

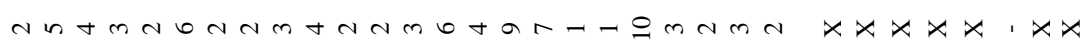

经

un $\backsim \backsim$ \&

尔

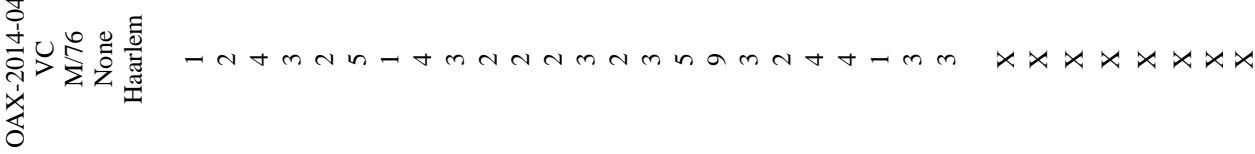

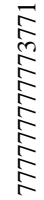

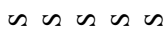

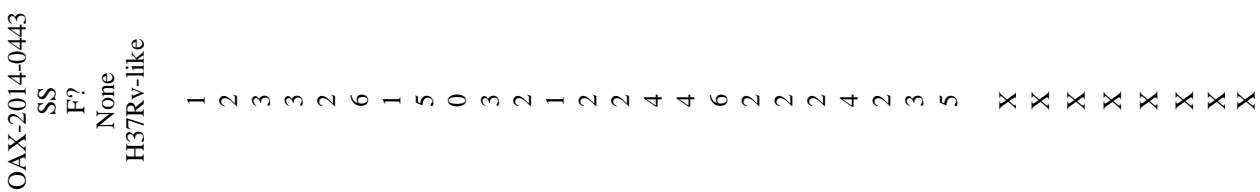




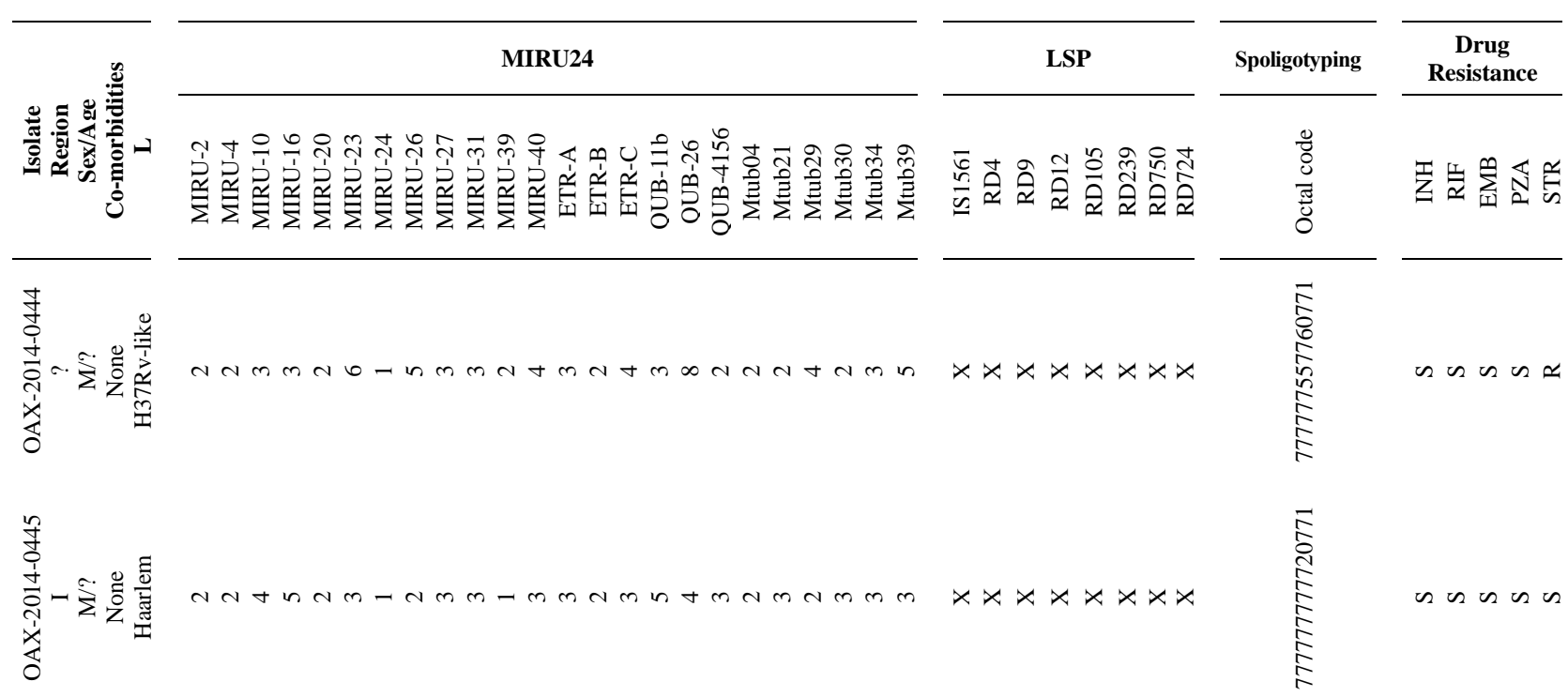

Region: C (Costa); I (Istmo); MIX (Mixteca); P (Papaloapan); SN (Sierra Norte); SS (Sierra Sur); VC (Valles Centrales) Sex/Age: M (Male); F (Female); ? (Unknown)

Co-morbidities: An (Anemia); Pn (Pneumonia); Mn (Malnutrition); DM (type 2 Diabetes mellitus); HTA (Arterial hypertension); HTP (Portal hypertension); HIV; Ci (Cirrhosis); HT (Hypothyroidism); Sm (Smoking); Og (Oligoclonal gamapathy); Ep (Epilepsy); Cs (Cannabis smoker); Ow (Overweight); Al

(Alcoholism); As (Asthma); None

L: Lineage

LSP: X (Present region); - (Absent region)

Drug resistance: INH (isoniazid); RIF (rifampicin); EMB (ethambutol); PZA (pyrazinamide); STR (streptomycin); S (sensitive); R(resistant)

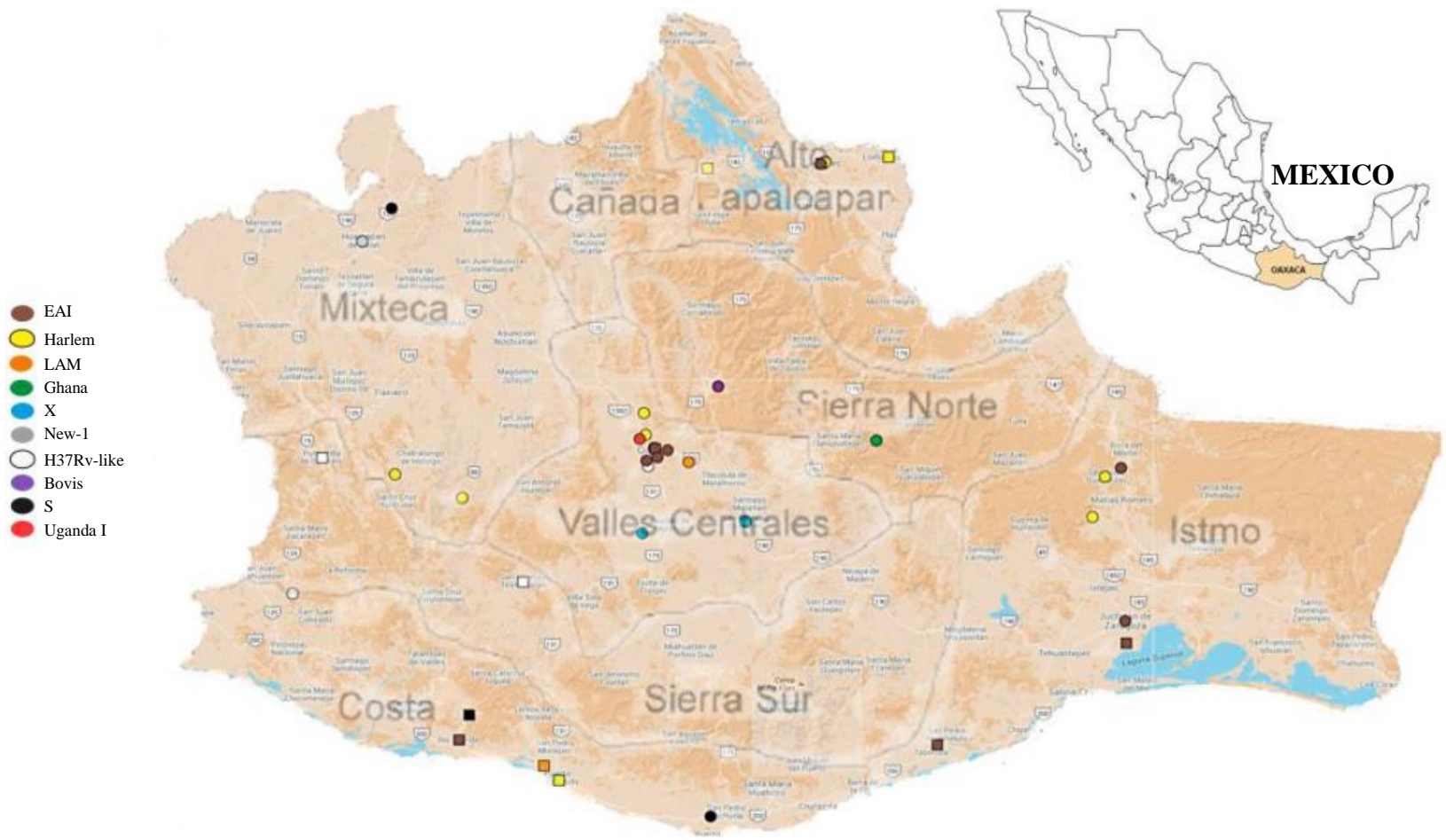

Fig. 2: Geographical localization of each sub-lineage and drug-resistance pattern within Oaxaca State, Mexico. Haarlem (yellow), EAI (brown), LAM (orange), Ghana (green), X (blue), New-1 (gray), H37Rv-like (white), M. bovis (purple), S (black) and Uganda I (red). Pan-susceptible isolates (circles) and isolates resistant to at least one first line drug (squares)

In the "Mixteca" and the "Papaloapan" regions, the Haarlem sub-lineage was predominant. However, in the
"Mixteca" region the isolates were susceptible to all first line anti-tuberculosis drugs, while in "Papaloapan" 
region, 66.6\% were drug-resistant. In the "Istmo de Tehuantepec" region, the EAI sub-lineage was the most frequent $(66.6 \%)$, showing a high incidence of first-line drug resistance $(50 \%)$. In the "Sierra Sur" region there were two isolates belonging to $\mathrm{H} 37 \mathrm{Rv}$-like sub-linage, one of them showed anti-tuberculosis drug resistance to RIF/PZA and the second to RIF/INH, considered as MDR-TB. Finally, in "Sierra Norte" region M. bovis was isolated from a 61 year-old woman.

\section{Discussion}

The genotypic diversity of clinical isolates in the state of Oaxaca differs from other national reports (Macias Parra et al., 2011; Martinez-Guarneros et al., 2013; Zenteno-Cuevas et al., 2013), specifically those from the northern and central region of the country (Lopez-Alvarez et al., 2010; Molina-Torres et al., 2010), where the genotypes T, LAM and $\mathrm{X}$ are the most frequently reported. The $\mathrm{T}$ lineage was not detected in the oaxacan population, besides LAM and X which occurred in low frequency, $12 \%$ and $10 \%$ respectively. Unexpectedly we detected a high occurrence of EAI sub-lineage $(26.1 \%)$, belonging to the Indo-Oceanic lineage, which has been reported at low frequencies throughout the country (Macias Parra et al., 2011; Molina-Torres et al., 2010), except for Acapulco City, in the state of Guerrero (Nava-Aguilera et al., 2011), where it was reported as the main genotype (44.6\%). It has been proposed that high occurrence of Indo-Oceanic strains could be due to their arrival directly from Philippines between XVI and XIX centuries when both countries were Spanish colonies and kept frequent maritime communication through the Pacific Ocean, where Oaxaca and Guerrero are located (Nava-Aguilera et al., 2011).

Similar to previous studies that report type 2 Diabetes Melltitus (DM) as one of the most frequent comorbidities in TB patients (Workneh et al., 2016), in this study it was present in $30.9 \%$ of the cases. Besides, TB-HIV coinfection has been reported between $28.8 \%$ and 64\% (Wejse et al., 2015; Middelkoop et al., 2015), here we found this binomial in just $11.9 \%$ of the patients. Haarlem sub-lineage predominates among DM patients (46\%). Similar results were found in another Mexican population study, where $53 \%$ of patients infected by Haarlem strains were diabetic (PérezNavarro, 2014). The EAI sub-lineage was the most frequent among the HIV co-infected patients (60\%), in concordance with another report in which this sublinage was present in $46.2 \%$ of TB-HIV coinfected patients (Kibiki et al., 2007).

In our study we observed a high percentage of primary resistance to at least one first line drug (35.71\%): MDR-TB (14.2\%), mono-resistant (14.2\%) and poly-resistant $(7.14 \%)$; being 1.5 times higher than in a previous drug resistance report from Oaxaca (Granich et al., 2000).

H37Rv-like sub-lineage was more associated with drug resistance $(60 \%)$ than the other lineages with two mono-resistant isolates to STR and RIF and one MDRTB isolate. To our knowledge there is only one report about drug resistance in this sub-lineage (Niemann et al., 2010), where no MDR-TB isolates were found.

The Haarlem sub-lineage has been linked with drugresistance and clonal expansion (Ramazanzadeh et al., 2015). We found that in "Papaloapan" region two of the three Haarlem isolates characterized were resistant to all drugs tested, in contrast with the isolates belonging to "Mixteca" region that were pan-susceptible. "Papaloapan" region is characterized by trade routes of agricultural products, is part of one of the migration routes from Central America to the USA and is better communicated with Veracruz than with Oaxaca. Reports about TB in Veracruz indicate that, together with Baja California, is the state with the highest prevalence of MDR-TB in the country (Juarez-Eusebio et al., 2017). So drug-resistant strains found in "Papaloapan" region may be associated with its geographical proximity to Veracruz. Furthermore, in the "Mixteca" region people prefer to use traditional medicine to treat diseases, in consequence they aren't exposed to drugs, which may not favour the development of drug-resistant strains.

There are some reports in which MDR-TB showed significant association with the EAI sub-lineage (Chen et al., 2017; Phyu et al., 2009). We observed that $45.5 \%$ of these strains were resistant to at least one first line drug: $9.1 \%$ monoresistant to RIF, $9.1 \%$ monoresistant to STR, 18.2\% MDR-TB and $9.1 \%$ polyresistant to INH/STR.

It has been demonstrated that Mexican diabetic patients present 4.7-fold and 3.5-fold higher risk to develop drug-resistance and MDR-TB, respectively (Pérez-Navarro et al., 2015). We found that among DM patients, $35.71 \%$ showed resistance to at least one drug and $7.14 \%$ was MDR-TB. In fact, in our study the most frequent comorbidities associated with drug-resistance were malnutrition (40\%) and HIV (40\%). Regarding to $\mathrm{HIV}$, there are reports of resistance to at least one drug that ranged from $10.6 \%$ in Tanzania (Kibiki et al., 2007), to $33.3 \%$ in Mexico City (Lopez-Alvarez et al., 2010), the latter more consistent with our results.

It is noteworthy that $16 \%$ of the analyzed isolates corresponded to NTM. This is an important percentage although lower than those previously reported for Mexico City (30.8\%) (Hernández-Solís et al., 2017) and Northern India (27.4\%) (Maurya et al., 2015). As in the above mentioned reports, we found $M$. avium, $M$. fortuitum and $M$. intracellulare as the most frequent infecting species. Now a days, AFB smear is still the preferred diagnosis method for TB, however it does not 
provide accurate information about the infecting specie, consequently, patients receive TB drug treatment which is not effective in NTM infections. It is extremely important that public health official policies include molecular techniques as part of the initial diagnosis in TB suspicious cases, in order to differentiate between MTBc and NTM. This methods may also provide drug resistance information and give faster results than mycobacterial isolation by culture.

This is a first approach to the description of the lineages circulating in Oaxaca and its association with drug resistance and co-morbidities. It is necessary to increase the number of isolates to draw wider conclusions. Although by molecular methods the detection of members belonging to MTBc was significant, it was not possible to recover all $\mathrm{AFB}(+)$ strains in culture; this probably due to the grueling journey to reach some communities, climate conditions and time of delivery to the laboratory. All of this circumstances may have affected the viability of the bacillus. Improving sample transport conditions should be considered in future studies.

\section{Conclusion}

According to this study, ten different MTBc sublineages were identified in Oaxaca, being Haarlem and EAI the most prevalent and related to patients with DM, HIV and malnutrition.

Drug resistance, including MDR-TB cases, was observed in isolates belonging to these two lineages, while most isolates form the rest of the identified sublineages were mainly pan-sensitive to all first line drugs.

The high diversity of sub-lineages found in "Valles Centrales" could be associated to the location of the Capital City in this region which implies migration, tourism and regional trading.

The identification of highly pathogenic MTBc genotypes and NTM in Oaxaca reveals the significance of implementing strategic surveillance molecular systems in order to identify possible outbreaks which may impact public health management in the state of Oaxaca.

\section{Acknowledgement}

We thankfully appreciate the technical assistance of Liria Pozos-Pineda, Sandra Isabel Espinoza-Castellanos and Luis Jesus Cortes-de-la-Fuente. We thank Clara Espitia Pinzon and Erika Segura Salinas from the Biomedical Research Institute of the National University of Mexico (Instituto de Investigaciones Biomedicas de la Universidad Nacional Autonoma de Mexico, UNAM) for providing TB reference strains DNA. We appreciate the support of Fidel Alejandro Sanchez Flores, from the Massive Sequencing and Bioinformatics Unit, Biotechnology Institute of the National University of
Mexico, on bioinformatics analysis. We specially thank Gabriela del Refugio Velazquez Rosas from Consejo Estatal para la Prevención y Control del Sida, for her authorization and support to this project. Finally, to Claudia Cruz Lescas, all state and jurisdictional TB program leaders in Oaxaca and all patients.

\section{Funding}

This work was supported by Consejo Nacional de Ciencia y Tecnología (CONACYT) and the Oaxaca State Government (FOMIX Grant number: 193298). ValenciaCarmona Oscar Daniel was a postgraduate student under CONACYT National Fellowship (404809). MartinezCruz Perla Monica is a postgraduate student under CONACYT National Fellowship (94467).

\section{Author's contribution}

Nakamura-Lopez, Yuko: Designed and supervised the study and contributed to data analysis.

Valencia-Carmona, Oscar Daniel: Conducted MIRU-VNTR and LPS experiments

Martinez-Cruz, Perla Monica: Data analysis and manuscript writing.

Palma-Nicolas, Jose Prisco: Conducted drug resistance and spoligotyping analysis.

Gonzalez-y-Merchand, Jorge Alberto: Consultant for genotyping (MIRU-VNTR).

Rivera-Gutierrez, Sandra: Technical support in genotyping.

Muñiz-Salazar, Raquel: Molecular confirmation of drug resistance.

Martinez-Martinez, Lucia Lourdes: Provided laboratory facilities and contributed to data analysis.

\section{Ethics}

All individuals included in this study, signed a written informed consent and answered a questionnaire to obtain socio-demographic and clinical data. The Ethical Committee on Investigation of the Consejo Estatal para la Prevencion y Control del Sida, OaxacaMexico, approved the protocol. As TB is a notifiable disease in Mexico, all confirmed TB cases were reported to the local public health authorities.

\section{References}

Allix, C., M. Fauville and P. Supply, 2008. Three-year population-based evaluation of standardized mycobacterial interspersed repetitive-unit-variablenumber tandem-repeat typing of Mycobacterium tuberculosis. J. Clin. Microbiol., 46: 1398-1406. DOI: 10.1128/JCM.02089-07 
Campos, S.B. and G.A. Flores, 1996. Manual de Procedimientos de Laboratorio INDRE/SAGAR: Tuberculosis. 1st Edn., Instituto Nacional de Diagnóstico y Referencia Epidemiológicos,

ISBN-13: 9688114952, pp: 100.

Chen, Y.Y., J.R. Chang, W.F. Huang, C.H. Hsu and H.Y. Cheng et al., 2017. Genetic diversity of the Mycobacterium tuberculosis East African-Indian family in three tropical Asian countries. J. Microbiol. Immunol. Infect., 50: 886-892. DOI: 10.1016/j.jmii.2015.10.012

Coll, F., R. McNerney, J.A. Guerra-Assuncao, J.R. Glyyn and J. Pedrigao et al., 2014. A robust SNP barcode for typing Mycobacterium tuberculosis complex strains. Nat. Commun., 1: 4812-4812.

DOI: $10.1038 /$ ncomms5812

Granich, R.M., M. Balandrano, A.J. Santaella, N.J. Binkin and K.G. Castro et al., 2000. Survey of drug resistance of Mycobacterium tuberculosis in 3 Mexican states 1997. Arch. Int. Med., 160: 639-644. DOI: 10.1001/archinte.160.5.639

Hernández-Solís, A., R. Cicero-Sabido, M. GonzálezVilla, I.I. Martínez-Rivera and A.D. MandujanoMartínez et al., 2017. Nontuberculous mycobacteria in clinical samples with negative acid-fast bacilli. Int. J. Mycobacteriol., 6: 391-395.

Huard, R.C., L.C. Lazzarini, W.R. Butler, D. van Soolingen and J.L. Ho, 2003. PCR-Based method to differentiate the subspecies of the Mycobacterium tuberculosis complex on the basis of genomic deletions. J. Clin. Microbiol., 41: 1637-1650. DOI: $10.1128 /$ jcm.41.4.1637-1650.2003

Juarez-Eusebio, D.M., D. Munro-Rojas, R. MunizSalazar, R. Laniado-Laborin and C.A. FloresLópez et al., 2017. Molecular characterization of multidrug-resistant Mycobacterium tuberculosis isolates from high prevalence tuberculosis states in Mexico. Infect. Genet. Evol., 55: 384-391.

DOI: 10.1016/j.meegid.2016.09.012

Kibiki, G.S., B. Mulder, W.M. Dolmans, J.L. de Beer and M. Boeree et al., 2007. M. tuberculosis genotypic diversity and drug susceptibility pattern in HIV-infected and non-HIV-infected patients in northern Tanzania. BMC Microbiol., 7: 51-51. DOI: 10.1186/1471-2180-7-51

Lopez-Alvarez, R., C. Badillo-Lopez, J.F. CernaCortes, I. Castillo-Ramirez and S. RiveraGutierrez et al., 2010. First insights into the genetic diversity of Mycobacterium tuberculosis isolates from HIV-infected Mexican patients and mutations causing multidrug resistance. BMC Microbiol., 10: 82-82.

DOI: $10.1186 / 1471-2180-10-82$
Macias Parra, M., J. Kumate Rodriguez, J.L. Arredondo Garcia, Y. Lopez-Vidal and M. Castanon-Arreola et al., 2011. Mycobacterium tuberculosis complex genotype diversity and drug resistance profiles in a pediatric population in Mexico. Tuberc Res. Treat.

Martinez-Guarneros, A., N. Rastogi, D. Couvin, A. Escobar-Gutierrez and L.M. Rossi et al., 2013. Genetic diversity among multidrug-resistant Mycobacterium tuberculosis strains in Mexico. Infect. Genet. Evol., 14: 434-443. DOI: 10.1016/j.meegid.2012.12.024

Maurya, A.K., V.L. Nag, S. Kant, R.A.S. Kushwaha and M. Kumar et al., 2015. Prevalence of nontuberculous mycobacteria among extrapulmonary tuberculosis cases in tertiary care centers in Northern India. BioMed. Res, Int., 2015: 465403-465403. DOI: 10.1155/2015/465403

Middelkoop, K., B. Mathema, L. Myer, E. Shashkina and A. Whitelaw et al., 2015. Transmission of tuberculosis in a South African community with a high prevalence of HIV infection. J. Infect. Dis., 211: 53-61. DOI: 10.1093/infdis/jiu403

Molina-Torres, C.A., E. Moreno-Torres, J. OcampoCandiani, A. Rendon and K. Blackwood et al., 2010. Mycobacterium tuberculosis spoligotypes in monterrey, Mexico. J. Clin. Microbiol., 48: 448-455. DOI: 10.1128/JCM.01043-14

Nava-Aguilera, E., Y. Lopez-Vidal, E. Harris, A. Morales-Perez and S. Mitchell et al., 2011. Clustering of Mycobacterium tuberculosis cases in Acapulco: Spoligotyping and risk factors. Clin. Dev. Immunol., 2011: 408375.

DOI: $10.1155 / 2011 / 408375$

Niemann, S., R. Diel, G. Khechinashvili, M. Gegia and N. Mdivani et al., 2010. Mycobacterium tuberculosis Beijing lineage favors the spread of multidrug-resistant tuberculosis in the Republic of Georgia. J. Clin. Microbiol., 48: 3544-3550.

DOI: 10.1128/JCM.00715-10

OPS/OMS, 2014. La tuberculosis en las Americas. OPS/OMS. www.paho.org/tuberculosis

Pérez-Navarro, L.M., F.J. Fuentes-Dominguez and R. Zenteno-Cuevas, 2015. Type 2 diabetes mellitus and its influence in the development of multidrug resistance tuberculosis in patients from southeastern Mexico. J. Diabetes Complic., 29: 77-82.

DOI: 10.1016/j.jdiacomp.2014.09.007

Pérez-Navarro, L.M., 2014. Caracterización Epidemiológico-Molecular de M. tuberculosis en Pacientes con Tuberculosis Pulmonar y Diabetes Mellitus del Estado de Veracruz. 1st Edn., Universidad Veracruzana, Xalapa, Veracruz, pp: 194. 
Petroff, S.A., 1915. A new and rapid method for the isolation and cultivation of tubercle bacilli directly from the sputum and feces. J. Exp. Med., 21: 38-42. DOI: $10.1084 /$ jem.21.1.38

Phyu, S., R. Stavrum, T. Lwin, O.S. Svendsen and T. Ti et al., 2009. Predominance of Mycobacterium tuberculosis EAI and Beijing lineages in Yangon, Myanmar. J. Clin. Microbiol., 47: 335-344. DOI: $10.1128 / \mathrm{JCM} .01812-08$

PUDI, 2014. Sistema Nacional de Vigilancia Epidemiologica (SINAVE). Secretaria de Salud. Mexico.

Ramazanzadeh, R., D. Roshani, P. Shakib and S. Rouhi, 2015. Prevalence and occurrence rate of Mycobacterium tuberculosis Haarlem family multidrug resistant in the worldwide population: A systematic review and meta-analysis. J. Res. Med. Sci., 20: 78-88.

Reed, M.B., V.K. Pichler, F. McIntosh, A. Mattia and A. Fallow et al., 2009. Major Mycobacterium tuberculosis lineages associate with patient country of origin. J. Clin. Microbiol., 47: 1119-1128.

DOI: $10.1128 / \mathrm{JCM} .02142-08$

Rindi, L., C. Medici, N. Bimbi, A. Buzzigoli and N. Lari et al., 2014. Genomic variability of Mycobacterium tuberculosis strains of the EuroAmerican lineage based on large sequence deletions and 15-locus MIRU-VNTR polymorphism. PLoS One, 9: e107150-e107150.

DOI: 10.1371/journal.pone.0107150
Supply, P., C. Allix, S. Lesjean, M. Cardozo-Oelemann and S. Rusch et al., 2006. Proposal for standarization of optimized mycobacterial interspersed repetitive unit-variable-number tandem repeat typing of Mycobacterium tuberculosis. J. Clin. Microbiol., 44: 4498-4510.

DOI: 10.1128/JCM.01392-06

Wejse, C., C.B. Patsche, A. Kuhle, F.J. Bamba and M.S. Mendes et al., 2015. Impact of HIV-1, HIV-2 and HIV $-1+2$ dual infection on the outcome of tuberculosis. Int. J. Infect. Dis., 32: 128-134.

DOI: $10.1016 /$ j.ijid.2014.12.015

WHO, 2015. Global Tuberculosis Report. 1st Edn., World Health Organization, ISBN-13: 97892415065059.

Workneh, M.H., G.A. Bjune and S.A. Yimer, 2016 Prevalence and associated factors of diabetes mellitus among tuberculosis patients in SouthEastern Amhara Region, Ethiopia: A cross sectional study. PLoS One, 11: e0147621-e0147621. DOI: 10.1371/journal.pone.0147621

Zenteno-Cuevas, R., F.X. Silva-Hernández, F. MendozaDamián, M.D. Ramírez-Hernández and K. VázquezMedina et al., 2013. Characterization of pks15/1 in clinical isolates of Mycobacterium tuberculosis from Mexico. Mem. Inst. Oswaldo Cruz, 108: 718-723. DOI: 10.1590/0074-0276108062013007 